\title{
Contraintes socioculturelles de la-non déclaration des naissances chez les communautés peulhs dans le Département de Korhogo (Côte d'Ivoire)
}

\author{
Kouamé Abou N'DRI, VANGA Adja Ferdinand et OURA Kan Constant \\ Université Péléforo Gon Coulibaly de Korhogo
}

\begin{abstract}
Despite the ratification of international laws on people's rights as well as those that regulate functioning of civil status in IVORY COAST, the problem of births declaration reveals intensively itself in certain communities especially among peulhs living in the Department of Korhogo. The aim of this study that is exclusively qualitative is to understand the social logics that explain non-declaration of births. At the end of the interviews with forty four persons through an investigation on the basis of saturation, the study shows that the phenomenon is due to the peulhs' perceptions about civil status and the increase of koranic education which creates a hostile environment to modernism. Moreover, the way of life based on the transhumance constitutes another justificative logic, for peulhs who consider that people doing transhumance don't need administrative documents because nature doesn't belongs to anyone.
\end{abstract}

Keywords:Culture, norms, non-déclaration of births, civil status, peulhs, Livestock farming, islam

\section{Résumé}

Malgré la ratification des lois internationales portant sur les droits des peuples ainsi que celles qui régissent le fonctionnement de l'état civil en Côte d'Ivoire, la question de la déclaration des naissances se pose avec acuité dans certaines communautés notamment chez les peulhs établis dans le Département de Korhogo. Cette étude purement qualitative a pour objectif de comprendre les contraintes socioculturelles qui soustendent la-non déclaration des naissances. Au terme des entretiens menés auprès de 44 enquêtés obtenus sur la base de la saturation, l'étude révèle que ce phénomène s'explique par les perceptions des peulhs de l'état civil ainsi que l'émergence de l'école coranique qui crée un environnement hostile au modernisme. De plus, le mode de vie caractérisé par la transhumance constitue une autre contrainte explicative d'autant plus que les peulhs estiment que le transhumant n'a pas besoin de documents administratifs car «la nature n'appartient à personne ».

Mots clés : Culture, normes, non déclaration des naissances, état civil, Peuls, élevage, Islam

\section{Introduction}

La déclaration des naissances à l'état civil est un acte indispensable pour la préservation des droits de l'enfant ainsi que sa protection contre l'apatridie (UNICEF, 2002). L'accession à l'emploi, aux logements, aux soins, aux transactions bancaires, à la légitimation des biens, aux services sociaux de base en dépendent (Ouafae, 2018). Elle est d'autant plus importante qu'elle constitue une obligation pour tout être humain vivant sur un espace territorial donné.

Cependant, en Côte d'Ivoire, 35\% des enfants de moins de 5 ans et 24\% des enfants de 5-17 ans n'existent pas légalement, faute d'avoir été enregistrés à l'état civil (EDSCI, 2012). Cette situation s'est aggravée pendant les 10 dernières années de crises surtout dans le nord du pays où il était devenu quasiment impossible, même aux parents qui le voudraient, de déclarer les naissances de leurs enfants à cause de 
l'effondrement de l'administration aux premières heures de la rébellion, en 2002. La crise postélectorale de 2010 rendra la tâche également difficile pour les parents dont les enfants sont nés entre novembre 2010 et juillet 2011. Dans la région du Gontougo par exemple, sur 21448 élèves au CP1, 48.86\% sont sans extraits (DRENA, 2012).

Face à ce phénomène qui constitue une atteinte aux droits des enfants, en 2008, le gouvernement et ses partenaires se sont accordés pour réformer le système d'état civil. Dès lors, la Côte d'Ivoire a fait de l'état civil un axe central de sa politique de protection des droits de l'enfant outre la loi $\mathrm{n}^{\circ}$ 64-374 du 7 octobre 1964 relative à l'état civil qui a été modifiée par la loi n 83-799 du 2 août 1983. Cette loi stipule que les naissances doivent être déclarées dans les trois (3) mois qui suivent l'accouchement. L'Etat ivoirien a aussi initié le 22 Mai 2019, le décret de loi portant dissolution de l'ONI et création de l'Office National de l'Etat Civil et de l'Identification de Côte d'Ivoire (ONECI). Ce décret de loi vise à rapprocher les structures de collectes des naissances des communautés notamment rurales. Dans cette perspective, des actions de terrains au profit de ces derniers en termes d'enregistrement des naissances ont été menées par l'Etat et ses partenaires au développement (OCHA, 2013).

Dans les zones CNO, plusieurs centres d'état civil endommagés ont été réhabilités après l'accord politique de Ouagadougou. Le département de Korhogo a bénéficié de cette attention des pouvoirs publics. Il a été créé des centres communautaires de collecte des naissances. Ainsi, à partir d'une approche communautaire, les populations locales ont été impliquées dans la chaine de collecte des naissances. Dans chaque village, un ou deux agents communautaires sont chargés de copter les naissances avant de les faire remonter aux centres de relai situés pour la plupart dans les villages; de sorte que ces centres transfèrent les dossiers locaux à la Sous-préfecture qui se charge de la délivrance des extraits de naissance. Les centres de santé ruraux sont également impliqués dans ce processus d'enregistrement des naissances dans la région du Poro. Les dossiers de naissances tels que constatés dans les structures sanitaires rurales sont alors transférés à la Souspréfecture afin que ces enfants soient enregistrés à l'état civil. Ce département a également bénéficié d'une opération qui a permis la régularisation de la situation administrative et juridique de 16.437 enfants sur un objectif de départ de 10.000 enfants sur 5 jours dans les communes et sous-préfectures de Korhogo, Niofoin, Sirasso, Tioro, Kanoroba, Komboro et Napié » a déclaré un des responsables du projet à Anadolu. Aussi, un système mobile de déclaration des naissances est mis en place pour faciliter l'opération .Ces dispositifs ont permis d'atteindre un taux de déclaration de naissance de 88, 4\% dans la région du Poro en 2017 (Annuaire statistique de l'état civil, 2017). Si de façon générale, ce taux connait une hausse en 2017, il a tout de même chuté en 2020, passant à 84,9\% (ASEC, 2020) du fait de la résistance de certaines communautés notamment les peulhs. En effet, Sur 84, 9\% de déclaration de naissance en 2020, la communauté peulhs totalise moins de cas d'enregistrement (Enquête exploratoire, 2020). Il existe, en effet, des normes qui alimentent leurs comportements face à la déclaration des naissances au point où leurs enfants sont pour la plupart légalement inconnus. Il est donc important de savoir les contraintes socioculturelles qui constituent des obstacles à la déclaration des naissances chez les communautés peulhs. Autrement dit, comment peut-on expliquer la-non déclaration des naissances chez les peulhs du Département de Korhogo ?

\section{Méthodologie}

Cette étude s'inscrit dans une approche qualitative. La collecte des données s'articule autour de la recherche documentaire et d'entretiens semi-directifs réalisés dans cinq (05) localités rurales du Département de Korhogo que sont Madinin,Nenekri, Koni, Kotcherie et Lataha(voir la carte). Le choix de ces villages se justifie par la forte présence des peulhs dans ces localités et surtout par le fait que ces derniers sont faiblement inscrits dans le registre de déclaration des naissances (Etat civil Korhogo). Au total, 44 personnes ont été entretenues comme l'indique le tableau ci-après:

Tableau 1 : Catégorie des personnes entretenues en fonction du genre

\begin{tabular}{|l|l|l|l|}
\hline Villages ou ville & Effectif & Hommes & Femmes \\
\hline Madinin & 11 & 07 & 04 \\
\hline Nenekri & 09 & 07 & 02 \\
\hline Koni & 06 & 04 & 02 \\
\hline
\end{tabular}




\begin{tabular}{|l|l|l|l|}
\hline Kotcherie & 10 & 06 & 04 \\
\hline Lataha & 08 & 05 & 03 \\
\hline Total & 44 & 29 & 15 \\
\hline
\end{tabular}

Source : (Enquête, 2021) Obtenu sur la base de la saturation

Les données recueillies à la suite de ce travail ont été retranscrites. Des analyses de contenus ont aussi été réalisées. Elles ont consisté à regrouper les informations en fonction des thématiques afin d'établir et d'analyser les rapports entre les différentes variables identifiées à partir de la triangulation des informations.

Carte : Présentation de la zone d'étude

\section{Source : Oura et Ouattara, 2020}

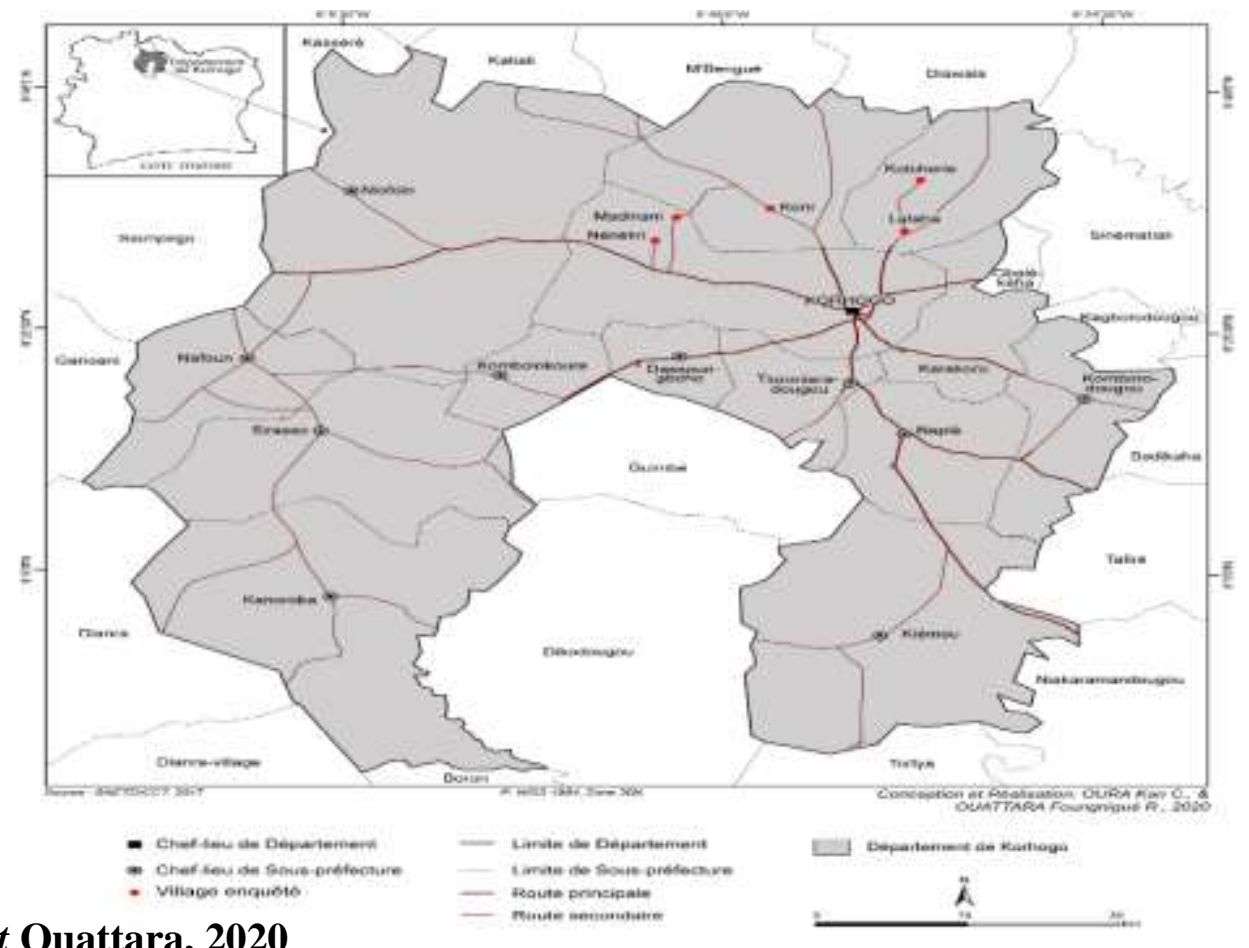

\section{Résultats}

L'étude révèle que les contraintes socioculturelles sont pour la plupart liées à des pesanteurs culturelles et religieuses marquées par une attitude égocentrique des peulhs qui ont tendance à sous-estimer les autres et surtout tout ce qui émane de la colonisation

\subsection{La primauté de la culture peulh, un frein à la déclaration des naissances}

La culture est le pilier de l'organisation sociale des communautés peulhs particulièrement celles du Département de Korhogo. Elle a une valeur suprême car chez les Peulhs, rien n'est au-dessus de cette culture. De ce fait, tout ce qui émane des pouvoirs publics occupe une place marginale dans les centres d'intérêts prioritaires de cette communauté. En fait, ils sont perçus comme un héritage colonial, donc opposés aux pratiques et normes de la vie sociale du peulh. Par exemple, l'extrait de naissance n'est rien d'autre, selon les Peulhs, qu'une forme de domination des peuples blancs sur les noirs. Par conséquent, il est inacceptable pour eux d'établir ce document assimilé à un symbole occidental comme le soutient cet enquêté :

«Chez nous, la culture est tout pour nous, elle prime sur tout. Tout ce que nous faisons, nous le faisons en fonction de nos valeurs et croyances culturelles. On ne peut pas laisser ce que nos parents nous ont appris pour faire autre chose surtout s'il s'agit du gouvernement qui copie tout sur les blancs. Nos parents n'ont jamais accepté leur présence en Afrique, nous non plus».

Ainsi, dans la conscience collective peulh, la priorité est accordée à la culture au point que ces normes culturelles créent un environnement hostile à la déclaration des naissances à l'état civil: 
«Avant, nos parents ne faisaient pas l'extrait des enfants puisqu'ils n'ont jamais accepté la présence des blancs. Aujourd'hui, nous aussi nous refusons de le faire pour la même raison. » (Propos de S. F un leader peulh de Lataha)

Cette perception de l'état civil par les Peulhs traverse les époques. Aujourd'hui, ces derniers s'en inspirer pour justifier leur réticence face à la déclaration des naissances à l'état civil.

Selon les peulhs, il n'est pas normal d'intégrer une communauté ayant acceptée la civilisation occidentale car cette attitude traduit la collaboration avec l'occident en raison du passé colonial du pays. Pour cela, ils ne jugent pas nécessaire de déclarer les naissances quoi que cela impacte fortement sur le niveau de scolarisation. En plus, la distance sociale entre peulhs et les tuteurs sociaux est devenue de plus en plus grande surtout avec leur activité de transhumance qui les isole davantage (Voir photo 1). Les tentatives de rapprochement connaissances parfois des échecs car en dépit des efforts faits par les autochtones, les peulhs restent toujours méfiants et égocentriques vis-à-vis de la communauté hôte.

Photo 1: Un campement peulh isolé, situé dans la localité de Madinin

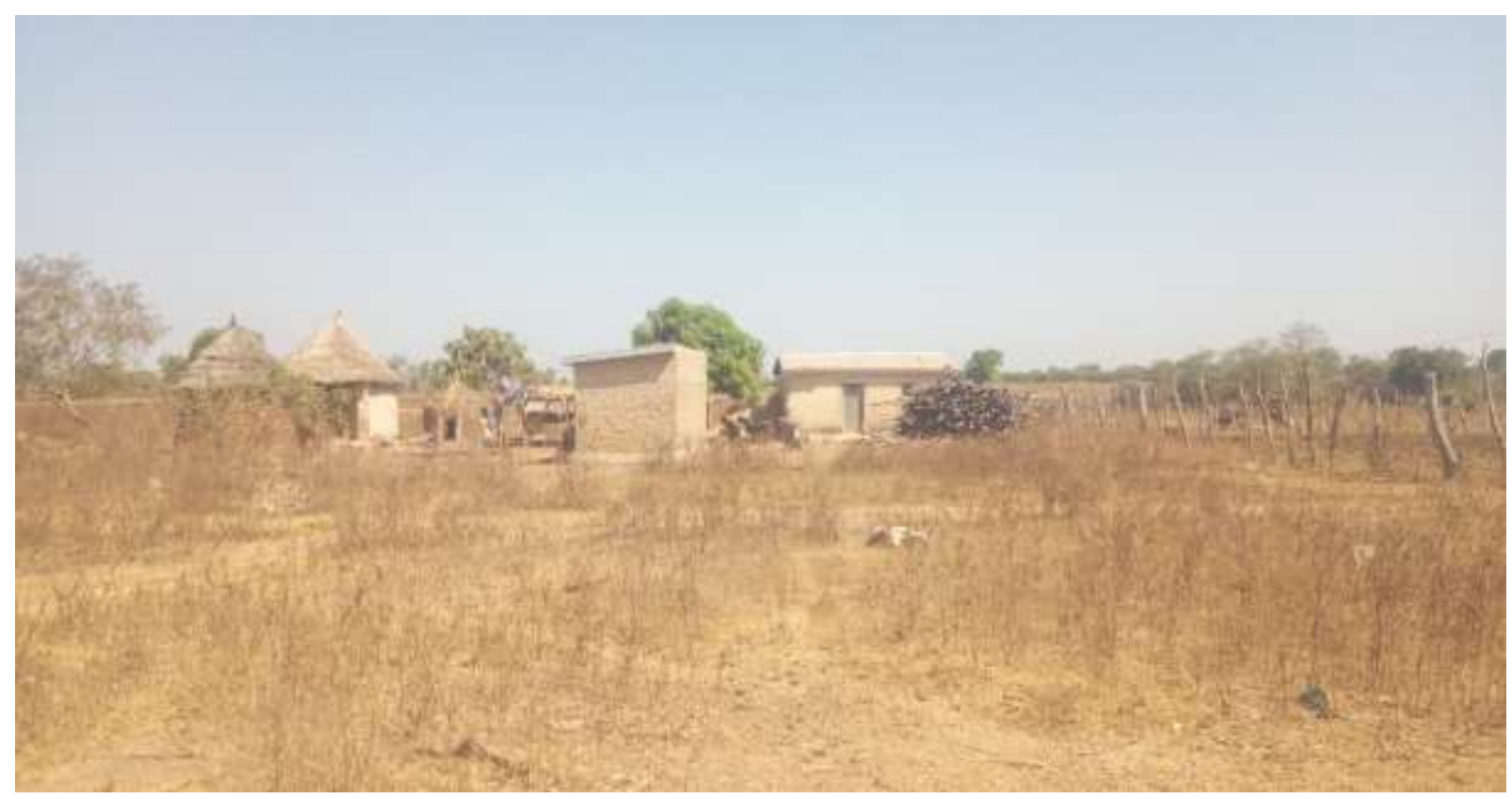

Source : Oura, Janvier 2020

Ainsi, le fait de vivre loin des tuteurs comme le traduit la photo ci-dessus est une stratégie d'éviter le contrôle de l'Etat par la négation de l'état civil car selon eux, «le peulh n'a pas de pays, il n'a donc pas besoin de papiers de l'Etat ».

\subsection{Les peulhs, un peuple sans frontière : La mobilité comme une identité culturelle}

Les Peulhs sont des communautés qui ont une tradition nomade. La mobilité est une identité culturelle. Pour ce faire, ils se déplacent de région en région, de pays en pays à la recherche des zones propices pour le développement de leurs bétails. Dans ce processus migratoire, ce peuple n'accorde aucune importance aux documents administratifs car, ces documents dans la conscience collective peulh, sont des moyens de contrôle de l'Etat sur les communautés. Or pour le peulh, les Etats contemporains sont perçus comme un héritage colonial dont les pratiques sont totalement contraires à la culture locale. Ainsi, dans ce contexte où le peulh ne croit qu'à ses propres valeurs, lorsqu'il s'agit de se déplacer, il dit n'avoir pas besoin d'être identifié car «la nature n'a pas de propriétaire, le peulh c'est le peulh», propos d'un tuteur peulh de Madinin. L'homme est libre de se déplacer et bénéficier des ressources de la terre. Il n'est donc pas question de déclarer les naissances à l'état civil comme l'explique un leader communautaire peulh de la localité de Nenekri : 
«Nous les Peulh, nous sommes un peuple indépendant. Partout où nous allons, nous affichons notre indépendance vis-à-vis des gens que nous trouvons sur place. La plupart d'entre nous ne font pas les papiers de leurs enfants pour ne pas dépendre du gouvernement ou avoir à vivre selon leurs conditions. Nos ancêtres ont toujours vécu de cette façon et nous également. Contrairement aux autres peuples qui vivent aujourd'hui selon les règles des blancs, le peulh n'a pas de pays. Il va partout où il veut».

Inspirés par le désir d'indépendance, les peulhs considèrent que la-non déclaration des naissances est comme une manière d'afficher leur autonomie face à un Etat qu'ils qualifient d'occidentalisé. Dans ce contexte d'occidentalisation des peuples, la communauté peulh refuse de briser un tabou culturel selon lequel :

«Quel que soit sa situation géographique, le peulh doit non seulement se démarquer des autres communautés mais aussi, éviter de s'acculturer. Lorsque nous les Peulhs, nous arrivons dans une localité, nous évitons de nous mélanger aux villageois pour ne pas avoir à vivre comme eux, pour ne pas qu'ils nous dominent ou nous imposent leur manière de vivre. C'est la même chose aussi pour le gouvernement. On évite de les approcher parce qu'il va prendre toutes sortes d'informations sur nous et nous dicter comment on doit vivre. Cela va nous amener à perdre notre culture comme les villageois qui sont ici. Le peulh ne répond qu'à sa culture ». (Propos d'un peulh de la Sous-préfecture de Korhogo)

Ces Propos montrent comment la culture amène les Peulhs à ne pas répondre aux exigences de l'Etat notamment en termes d'identification. Ils s'y opposent parce que cela peut être source d'acculturation et d'obstacles à la préservation de leur culture et au renouvellement générationnel. La déclaration des naissances à l'état civil n'est pas une nécessité d'autant que les fondements culturels y sont opposés. Aussi, les croyances religieuses auxquelles sont attachées les Peulh c'est-à-dire l'islam n'inscrit pas les documents administratifs au cœur du processus d'apprentissage.

\subsection{Du processus de transfert intergénérationnel de l'islam en société peulhs à la-non déclaration des naissances à l'état civil}

L'islam est la principale religion pratiquée par les communautés peulhs du Département de Korhogo. Cet ancrage de la religion islamique au sein des sociétés peulhs prend ses fondements dans la culture. Les Peulh pratiquent l'islam depuis des générations au point où il est aujourd'hui perçu par ces derniers comme une identité culturelle.

«L'islam, c'est notre culture, c'est une partie de nous. Nous ne pouvons le laisser au profit d'une autre religion. » (Propos d'un peulh de Koni)

Fortement attachés à l'islam, les peulhs envoient leurs enfants dans les écoles coraniques pour assurer la continuité de cette religion perçue comme un héritage qui doit se transmettre de génération en génération. Dès le bas âge, généralement à partir de cinq ans, les parents inscrivent leurs enfants dans ces écoles où il le confie directement à un dignitaire religieux afin que celui-ci se charge de son éducation. En outre, les parents eux-mêmes interviennent aussi dans ce processus comme l'explique un peulh de Lataha :

«Dès l'âge de 5 ans, j'apprends à mon enfant à prendre l'ablution et à faire la prière. Je le fais parce que mon enfant doit absolument être un musulman. Je ne peux pas imaginer mon enfant pratiquer une autre religion que l'islam. ».

Le but, à travers cette formation, est de permettre à ces adeptes de maitriser les techniques de la prière islamique et de mémoriser le coran afin d'éviter d'être des enfants sans repère social. Ce choix s'explique par le fait que l'islam a un ancrage culturel contrairement à l'école conventionnelle que les Peulhs qualifient d'«héritage colonial». Durant la formation, les maîtres coraniques se concentrent uniquement sur l'enseignement du rapport à «Dieu », à la société, au travail qui en réalité ne nécessite pas de documents administratifs comme le soutient C. P de Nenekri 
«Nous mêmes, nous ne comprenons pas pourquoi il ne nous demande pas de faire l'extrait des enfants. Les enfants vont tous à l'école coranique mais aucun n'a de papier. Aussi, je ne sais pas ce que ce papier va permettre à mon enfant de faire là-bas ». Déclare un peulh de la Souspréfecture de Korhogo.

En réalité, les Peulh ont une méconnaissance de l'importance de l'extrait de naissance. Mais avec l'émergence de l'islam dans cette communauté, l'implication des leaders religieux dans le processus de déclaration des naissances à l'état civil aurait pu être bénéfique pour l'Etat dans ses campagnes de sensibilisation malgré l'attachement des peulhs à la transhumance du fait de leur activité économique, l'élevage.

\subsection{La transhumance, une entrave à la déclaration des naissances à l'état civil}

La transhumance se définit comme le déplacement saisonnier d'un troupeau en vue de rejoindre une zone où il pourra se nourrir. Ce déplacement est le fondement du mode de vie de la communauté peulh dont l'activité principale est l'élevage. Dans cette communauté, l'existence sociale et culturelle en dépend. Cette activité est alors déterminante dans la classification sociale car elle permet d'accéder à une fonction sociale mais aussi à l'estime de la communauté comme le dit D. F de la communauté peulh de Lataha:

"L'élevage, c'est notre culture. C'est l'activité de nos ancêtres et c'est ce qu'ils nous ont appris à faire. Tout bon peulh doit savoir le faire et avoir ses boufs. C'est à travers la taille du troupeau qu'on distingue le riche du pauvre. C'est par la taille du troupeau qu'on choisit aussi nos chefs et autres responsables communautaires ».

En fait, renoncer à la pratique de l'élevage induit l'exclusion sociale du peulh par sa communauté. Comme conséquence, il est marginalisé et rejeté. Il est aussi exempté de toute fonction et de toute participation aux prises de décisions. Sa lignée est de ce fait victime d'un racisme «communautaire » comme le soutient $\mathrm{S}$. A. de la communauté peulh de Nenekri :

«Chez nous, si tu n'as pas de bœufs, tu n'es rien. On ne te considère pas et même tous tes descendants ».

En guise d'une conformité avec le mode de vie ancestral, les peulhs, même si certains parmi eux, avec la modernité, exercent d'autres métiers tels que le commerce, la plupart est restée fidèle à la pratique de l'élevage malgré les contraintes. Ces éleveurs passent la majeure partie de leur temps dans la nature en marge de la société hôte et de l'administration au point où ils méconnaissent la nécessité des documents administratifs dont l'extrait de naissance comme le dit D. S un responsable peulh de Kotcherie:

«Nous sommes toujours en brousse avec les animaux, qu'est-ce que l'extrait peut permettre à l'enfant de faire là-bas? Même si on fait, il ne va pas s'en servir donc cela ne vaut pas la peine ». Déclare un peulh de Kotcherie

Dans l'imaginaire des éleveurs peulhs du Département de Korhogo, l'extrait de naissance est uniquement utile pour les enfants du milieu urbain comme le soutient B. A de la communauté peulh de Madinin :

«On n’a pas besoin de papier pour être dans la nature avec les bæufs ».

Selon les tuteurs sociaux des peulhs, les campagnes de sensibilisation menées auprès de cette communauté concernant la régularisation de leur situation administrative. Elles se heurtent à des considérations idéologiques et culturelles. En fin de compte, le dialogue social est rompu car selon un chef de la communauté senoufo :

"Le peulh est difficile car ce qu'il pense c'est ce qu'il veut entendre ».

En outre, la non-déclaration des naissances chez les Peulhs est une stratégie pour éviter de se faire fichier et payer les taxes d'Etat car la majorité ne possède pas les pièces afférentes à leur activité. De ce fait, ils s'éloignent de l'administration afin d'éviter qu'ils soient identifiés. Ainsi, au fil du temps, la crainte de l'Etat 
dévient de plus en plus grande au point d'impacter négativement les rapports entre les autorités en charge de l'Etat civil et ces communautés peulhs. C'est d'ailleurs ce qui ressort des propos de S. K du village de Neninkri.

«Les peulhs ont peur de faire les papiers de leurs enfants. Comme ils n'ont pas de papiers, il est difficile pour eux d'aller voir le commandant. Il peut demander à vérifier leurs papiers. Il peut les mettre en prison ou encore les faire retourner au Burkina».

Par ailleurs, lors des périodes de transhumance, les peulhs s'installent en périphérie des villages tuteurs afin d'éviter des chocs culturels. Cette rupture du lien social entre les tuteurs sociaux et les Peulh a des implications sociales notamment l'absence de communication et de plateforme d'échanges entre les communautés. En fin de compte, ces peulhs évoluent dans un environnement où ils sont hors de l'Etat et ses principes de fonctionnement. Loin d'être néfastes pour elles, ces communautés au contraire se sentent plus épanouir dans cet environnement où la seule contrainte qui guide leur vie est la tradition car disent-elles :

«Nous préférons vivre comme ça parce que nous ne voulons pas être dérangé par des problèmes de papier qui en réalité ne nous servent à rien. Moi je travaille et je vis en brousse. ».

En claire, leur mode de vie, caractérisé par la transhumance influence négativement leurs rapports à l'état civil et aux tuteurs sociaux. En conséquence, ils développent en eux un rejet de l'Etat et de ses valeurs d'autant plus qu'ils estiment ne pas bénéficier de ces privilèges comme le soutient une autorité de la ville de Korhogo :

«Au cours de nos tournées de sensibilisation sur l'utilité de la déclaration des naissances, il ressort très souvent que les peulhs voudraient savoir si la déclaration des naissances leur conférait le droit à la nationalité ivoirienne. Quand nous leur disons non, alors ils nous répondent que l'extrait n'est pas important pour eux».

Selon les Peulhs enquêtés, l'Etat ne mène pas d'actions efficaces pour lutter contre les conflits qui les opposent aux agriculteurs. De ce fait, ils estiment qu'ils sont marginalisés dans la mesure où l'Etat ne leur apporte pas assistance.

«Le papier qu'on nous demande de faire pour nos enfants là, est inutile. Nous sommes très souvent en conflit avec les villageois et chaque fois il y a des gens qui meurent ou sont blessés ou même on perd des animaux. Ils ne font rien pour nous aider. Le papier qu'on fait pour les enfants là, c'est pour quoi alors? Je ne veux pas de leur papier pour mon enfant », Propos d'un peulh de Madinin.

L'attitude des peulhs face à l'état civil est une forme de protestation contre l'Etat dans la gestion des conflits éleveurs-agriculteurs récurrents dans le nord de la Côte d'Ivoire surtout le mode de règlement des conflits qui les opposent généralement aux tuteurs sociaux.

\section{Discussion}

Le profond attachement du peulh à sa culture est un handicap à son intégration aux changements que subit la société au point de rejeter tout ce qu'il juge être le produit de la colonisation. C'est le cas par exemple des déclarations des naissances à l'état civil des enfants issus de ces communautés comme l'indiquent les résultats de cette étude. Pour ce peuple, l'état émane d'un héritage colonial. De ce fait, il serait une forme de domination de l'occident sur les peuples noirs comme le soutient un rapport de l'UNICEF. En effet, des communautés développent une réticence vis-à-vis de l'état civil parce qu'elles estiment que l'état civil est le fruit d'une collaboration entre l'Etat et l'occident et donc elles l'associent à l'identité occidentale. Au Kenya par exemple, des communautés présentent l'état civil comme un héritage de la colonisation. De ce fait, il n'est pas question pour elles de déclarer leur naissance (UNICEF, 2002).

Outre cela, l'état civil est perçu par le peulh comme un moyen de contrôle de l'Etat. Il se sert des données pour assoir sa domination. Cette idée est en contradiction avec les résultats d'une étude commanditée par 
l'UNICEF qui montrent les fondements réels de la déclaration des naissances. Il s'agit plutôt de permettre à chaque personne de bénéficier de ses droits et sa prise en compte dans les programmes de développement. L'état civil évite aussi que l'enfant devienne plus tard un apatride. En fait, sans identité, il ne peut ni voyager, ni bénéficier de ses droits puisqu'il n'existe pas (UNICEF, Op. Cit.). Quant à Vircoulon (2017), il associe la-non déclaration des naissances à une forme d'ignorance sociale des populations de l'importance de l'état civil comme l'indique les résultats de l'étude. En effet, la transhumance bovine contraint les éleveurs peulhs à passer la majeure partie de leur temps dans la nature. Ce mode de vie les amène à vivre en marge de la société au point où ils ne perçoivent pas en quoi l'extrait pourrait servir au développement d'un pays, à un enfant.

Par ailleurs, l'islam, la principale religion des Peulhs, constitue un obstacle à la déclaration des naissances à l'état civil. Le problème se situe dans l'orientation éducative et le processus d'islamisation. Ils estiment que c'est un devoir pour eux envers leur communauté et la religion de convertir leur progéniture à l'islam. Les enfants sont, de ce fait, inscrits dans ces écoles ou confiés à des prédicateurs. Cette éducation religieuse se fait, cependant, au détriment de l'école conventionnelle. Contrairement au système éducatif classique où l'extrait de naissances est exigé, l'enseignement coranique met plutôt l'accent sur la connaissance islamique. L'extrait de naissance ne faisant pas parti des priorités de la formation comme le soutiennent YEO et al (2017). Ces auteurs ont montré qu'il existe un lien étroit entre l'islam, la déscolarisation et la-non déclaration des naissances peulhs à l'état civil. Pour eux, le fait que les parents peulhs inscrivent les enfants à l'école coranique, est un facteur d'ignorance de l'importance de l'état civil. Quant à Vircoulon (2017), il établit un lien entre l'activité des peulhs et la connaissance de l'état civil. Pour lui, la-non déclaration des naissances des enfants peulhs serait le résultat de la mobilité de ces éleveurs comme l'atteste les résultats de cette étude. Ils sont à la recherche permanente d'endroits propices à l'élevage. Cette mobilité a pour conséquence la méconnaissance du système d'état civil car durant les campagnes de transhumance, ils installent généralement leurs campements loin des villages hôtes afin d'éviter les conflits entre eux et leurs tuteurs sociaux (Bernardet, 1986). En fait, selon ces communautés, l'Etat est toujours resté indifférent face à ces conflits comme le soutient Catherine (2010) lorsqu'elle évoque la question de protection des Roms en France qui vivent en marge de la société française. Aussi, les Peulhs s'organisent et passent la plupart de leur temps en compagnie du bétail dans la nature car comme l'atteste Diallo (1995), la richesse d'un peulh est fonction de son cheptel, car l'estime de sa communauté en dépend comme le révèlent les résultats de l'étude.

\section{Conclusion}

Le présent article analyse les contraintes socioculturelles de la-non déclaration des naissances particulièrement chez les communautés peulhs du Département de Korhogo. A partir d'une approche qualitative, l'étude révèle que la culture et la religion sont le fondement de la-non déclaration des naissances chez les peulhs car ces derniers assimilent l'état civil à un héritage colonial. Ainsi, les préjugés autour de l'état civil traversent toutes les générations au point que cette communauté est devenue réfractaire à la plupart des décisions qui sont prises par les pouvoirs publics. En fin de compte, l'attitude de cette communauté a pour conséquences l'émergence d'une catégorie d'enfants apatrides qui pour la plupart sont privés de leurs droits élémentaires tels que l'accès aux services sociaux de base notamment l'éducation, la santé, l'extrait de naissance.

\section{Références bibliographiques}

1. Bernardet, P. (1986), Éleveurs et agriculteurs en Côte d'Ivoire : spécialisation et complémentarité. In Blanc-Pamard Chantal (ed.), Boutrais Jean (ed.) Dynamique des systèmes agraires : à la croisée des parcours : pasteurs, éleveurs, cultivateurs.ORSTOM(Paris), pp. 237-268. ISBN 2-7099-1228-7 ISSN 0767-2896.

2. Catherine, A.C., (2010); Le droit français et la protection des Roms. Cahiers de la recherche sur les droits fondamentaux, 8 . 
3. CODE CIVIL I. (2019). Droit des biens et des obligations, Journal officiel

4. Diallo, I., (2010). Les pasteurs nomades au Burkina-Faso. Pasteurs nomades et transhumances autochtone, Paris, Harmattan.

5. OCHA., (2013). Bulletin humanitaire numéro 07.

6. Ouafae, S. (2018). Les enfants fantômes : un défi pour la réalisation des ODD. [Conférence] Sensibilisation des Etats et information des populations sur l'importance de l'état civil.https://www.etatcivil.pw-content/upoads/sites/5/2018/09Note-Synthèse-Ouafae-Sananes-ADFLes-enfants-fantomes-15-juin-2018.pdf

7. UNICEF, (2002). L'enregistrement à la naissance : un droit pour commencer, Florence: centre de recherche international.

8. Vircoulon, T. (2017). Etat civil en République centrafricaine : enjeux et pratiques.https://www.asf.be/wpcontent/uploads/2017/08/ASF_RCA_EtatCivil_201707_FR.pdf

9. YEO et al., (2017). Étude des déterminants de non-déclaration des enfants scolarisés à l'état civil en côte d'ivoire. NODUS SCIENDI, 22. 